\title{
Consistency of linkage disequilibrium between Chinese and Nordic Holsteins and genomic prediction for Chinese Holsteins using a joint reference population
}

\author{
Lei Zhou', Xiangdong Ding ${ }^{2 *}$, Qin Zhang ${ }^{2}$, Yachun Wang ${ }^{2}$, Mogens S Lund ${ }^{1}$ and Guosheng Su ${ }^{\text {** }}$
}

\begin{abstract}
Background: In China, the reference population of genotyped Holstein cattle is relatively small with to date, 80 bulls and 2091 cows genotyped with the Illumina 54 K chip. Including genotyped Holstein cattle from other countries in the reference population could improve the accuracy of genomic prediction of the Chinese Holstein population. This study investigated the consistency of linkage disequilibrium between adjacent markers between the Chinese and Nordic Holstein populations, and compared the reliability of genomic predictions based on the Chinese reference population only or the combined Chinese and Nordic reference populations.
\end{abstract}

Methods: Genomic estimated breeding values of Chinese Holstein cattle were predicted using a single-trait GBLUP model based on the Chinese reference dataset, and using a two-trait GBLUP model based on a joint reference dataset that included both the Chinese and Nordic Holstein data.

Results: The extent of linkage disequilibrium was similar in the Chinese and Nordic Holstein populations and the consistency of linkage disequilibrium between the two populations was very high, with a correlation of 0.97. Genomic prediction using the joint versus the Chinese reference dataset increased reliabilities of genomic predictions of Chinese Holstein bulls in the test data from 0.22, 0.15 and 0.11 to $0.51,0.47$ and 0.36 for milk yield, fat yield and protein yield, respectively. Using five-fold cross-validation, reliabilities of genomic predictions of Chinese cows increased from $0.15,0.12$ and 0.15 to $0.26,0.17$ and 0.20 for milk yield, fat yield and protein yield, respectively.

Conclusions: The linkage disequilibrium between the two populations was very consistent and using the combined Nordic and Chinese reference dataset substantially increased reliabilities of genomic predictions for Chinese Holstein cattle.

\section{Background}

Genomic selection was proposed in 2001 [1] and has since then become a major research topic in animal breeding. Accuracy of genomic prediction depends greatly on the size of the reference population $[2,3]$. The larger the reference population, the more accurate genomic prediction is. It was reported that reliabilities of

\footnotetext{
* Correspondence: xding@cau.edu.cn; guosheng.su@agrsci.dk ${ }^{2}$ College of Animal Science and Technology, China Agricultural University, Beijing 100193, China

'Department of Molecular Biology and Genetics, Aarhus University, Tjele DK8830, Denmark

Full list of author information is available at the end of the article
}

genomic prediction of Holstein cattle increased when Holstein cattle of other countries were added to the reference dataset [4-6]. Similarly, pooling genotypes from other countries or populations to form a common reference population helped to increase the reliability of predictions in Brown Swiss cattle [6,7]. In addition, reliabilities of genomic prediction obtained by combining the reference populations of Danish, Swedish and Finnish red cattle were higher than those using singlecountry reference populations [8]. Holstein dairy cattle in China were originally imported from Europe and North America and were mostly derived from crossbreeding between the local yellow cattle and imported foreign Holstein bulls. It is assumed that the current 
Chinese Holstein population is genetically close to the other Holstein populations in the world. To date, the reference population of genotyped Holstein cattle in China is relatively small and includes mainly cows. It is expected that a joint reference dataset that combines Chinese Holstein cattle and Holstein cattle from other populations will greatly improve the reliability of genomic predictions of the Chinese Holstein population, assuming linkage disequilibrium between markers and quantitative trait loci (QTL) is consistent between the populations.

The objectives of this study were to: (1) estimate the consistency of linkage disequilibrium between the Chinese and the Nordic Holstein populations and (2) assess the gains in reliability of genomic predictions in Chinese Holstein from using a joint Chinese and Nordic reference dataset, compared with using the Chinese reference dataset alone.

\section{Methods \\ Data}

In this study, both the Chinese Holstein $(\mathrm{CH})$ and Nordic Holstein $(\mathrm{NH})$ cattle were genotyped with the Illumina BovineSNP50 BeadChip (Illumina, San Diego, $\mathrm{CA}$ ). The single nucleotide polymorphism (SNP) data of each population were edited separately by deleting SNP with minor allele frequencies less than 0.01 or call rates less than 0.10 , and excluding individuals with more than 10\% missing marker genotypes. After editing, 41838 SNP on 29 autosomes were retained in both populations. The genotyped $\mathrm{CH}$ cattle included 80 bulls born between 1993 and 2002 and 2091 cows born between 2001 and 2006, which were daughters of 13 of the genotyped bulls. The number of daughters per bull ranged from 63 to 358, with a mean of 135. The genotyped NH cattle included 5216 bulls born between 1974 and 2008. All animals of both populations were used in the linkage disequilibrium analysis. Deregressed proofs (DRP) were used as phenotypes for genomic prediction. DRP of $\mathrm{CH}$ bulls and cows were derived from the estimated breeding values (EBV) obtained from the Chinese genetic evaluations in April 2012 (Dairy Association of China), and DRP of NH bulls were derived from the EBV of Nordic genetic evaluations in November 2010 (Nordic Genetic Evaluation). Three traits (milk yield, fat yield and protein yield) were analyzed. In total, $4398 \mathrm{NH}$ bulls and all $\mathrm{CH}$ animals had phenotypes for the three traits. $512 \mathrm{CH}$ cows with possible incorrect sire information were discarded based on parentage verification with 255 SNP performed in a previous study [9] in which paternity was considered incorrect if five or more SNP were in conflict (i.e., a sire was homozygous for one allele but its daughter was homozygous for the other allele). Consequently, 1572 $\mathrm{CH}$ cows and $80 \mathrm{CH}$ bulls with reliable pedigree information were used for genomic prediction.

\section{Measure of linkage disequilibrium (LD) and consistency of LD}

Each chromosome was phased separately using Beagle [10] for each population, and all missing genotypes were simultaneously imputed by Beagle. Linkage disequilibrium between a pair of SNP was measured as $r_{L D}^{2}$ [11], and $r_{L D}$ was calculated as follows

$$
r_{L D}=\frac{f(A B)-f(A) f(B)}{\sqrt{f(A) f(a) f(B) f(b)}},
$$

where $f(A B), f(A), f(a), f(B)$ and $f(b)$ are observed frequencies of haplotype $\mathrm{AB}$, alleles $\mathrm{A}, \mathrm{a}, \mathrm{B}$ and $\mathrm{b}$, respectively. Maternal and paternal haplotypes were pooled to calculate LD. The consistency of LD in the two populations was measured by the correlation of $r_{L D}$ of adjacent marker pairs on each autosome between the two populations [12].

\section{Prediction of genomic breeding values}

A single-trait GBLUP model was used for genomic prediction based on the Chinese reference dataset, and a two-trait GBLUP was used for genomic prediction based on the joint reference dataset that included both the $\mathrm{CH}$ and $\mathrm{NH}$ cattle. In the latter, a single biological trait was regarded as a different trait in the two populations. The basic GBLUP model $[13,14]$ was

$$
\mathbf{y}=\mathbf{1} \mu+\mathbf{Z g}+\mathbf{e}
$$

where $\mathbf{y}$ is the vector of phenotypes, $\mu$ is the population mean, $\mathbf{g}$ is the vector of breeding values, $\mathbf{e}$ is the vector of residuals, and $\mathbf{Z}$ is a design matrix allocating $\mathbf{g}$ to $\mathbf{y}$. It was assumed that $\mathbf{g} \sim N\left(\mathbf{0}, \mathbf{G} \sigma_{g}^{2}\right)$ and $\mathbf{e} \sim N\left(\mathbf{0}, \mathbf{D} \sigma_{e}^{2}\right)$, where $\mathbf{G}$ is the genomic relationship matrix, $\sigma_{g}^{2}$ is the additive genetic variance, $\mathbf{D}$ is a diagonal matrix with weights of the residual variance [15], and $\sigma_{e}^{2}$ is the residual variance. The $\mathbf{G}$ matrix was constructed according to the method (method 1) described by VanRaden [14], i.e. $\mathbf{G}=\mathbf{M} \mathbf{M}^{\prime} /$ $\sum 2 p_{i}\left(1-p_{i}\right)$, where elements in column i of $\mathbf{M}$ are $0-2 \mathrm{p}_{\mathrm{i}}$, $1-2 p_{i}$ and $2-2 p_{i}$ for genotypes $A_{1} A_{1}, A_{1} A_{2}$ and $A_{2} A_{2}$, respectively, and $p_{i}$ is the allele frequency of $A_{2}$ at locus $i$, calculated from the available marker data. Variances and covariances in the GBLUP models were estimated using the "average information" restricted maximum likelihood algorithm, and the GBLUP analyses were conducted using the DMU Package [16]. 


\section{Validation}

Accuracy of genomic predictions using the $\mathrm{CH}$ and the joint reference datasets was assessed by two validation procedures. In the first procedure, the reference dataset comprised 13 genotyped $\mathrm{CH}$ bulls and their genotyped daughters and the test dataset consisted of $48 \mathrm{CH}$ bulls without genotyped daughters. The remaining $19 \mathrm{CH}$ bulls, which were highly related with the 13 reference bulls, were not used in the test dataset. In the second procedure, a five-fold cross-validation procedure was used for genomic prediction of $\mathrm{CH}$ cows. In each fold of cross-validation, two or three half-sib families of cows were used as the test dataset and the remaining cows and genotyped $\mathrm{CH}$ bulls were used as reference dataset. The numbers of animals in the reference and test datasets for the two validation procedures are shown in Table 1. Genomic EBV (GEBV) for the animals in a test dataset were predicted using both the $\mathrm{CH}$ and the joint reference datasets. The joint reference dataset included all 4398 Nordic bulls.

Reliabilities of GEBV for bulls and cows were measured as the squared correlation of GEBV and DRP divided by the average reliabilities of the DRP in the test dataset $\left(\operatorname{Cor}_{(\mathrm{GEBV}, \mathrm{DRP})}^{2} / \mathrm{r}_{\mathrm{DRP}}^{2}\right)$ [15]. Because $\mathrm{CH}$ bulls were born between 1993 and 2002, genetic trend due to selection could inflate the correlation between GEBV and DRP. Therefore, in the validation of genomic predictions for $\mathrm{CH}$ bulls, genetic trends present in GEBV and DRP were corrected by regressing on birth year, and then the reliabilities were calculated by correlating the corrected GEBV and DRP. In the validation of genomic predictions for cows, reliabilities were calculated based on GEBV pooled over the five test datasets.

\section{Results and discussion}

\section{Linkage disequilibrium and consistency of LD}

Data on the 41838 SNP distributed over the 29 bovine autosomes are summarized in Table 2. The average distance between adjacent SNP was $59.59 \mathrm{~Kb}$, and the shortest and longest gaps were 3 bp (on BTA7) and 3820

Table 1 The size of test and reference datasets used for validating genomic predictions of Chinese Holstein (CH) bulls and cows

\begin{tabular}{llll}
\hline Categories & \multicolumn{2}{c}{ Test datasets } & Reference datasets \\
\hline CH bulls & \multicolumn{2}{c}{48} & 13 bulls and 1572 cows \\
\hline CH cows & set 1 & 337 & 80 bulls and 1235 cows \\
\cline { 2 - 4 } & set 2 & 309 & 80 bulls and 1263 cows \\
\cline { 2 - 4 } & set 3 & 323 & 80 bulls and 1249 cows \\
\cline { 2 - 4 } & set 4 & 307 & 80 bulls and 1265 cows \\
\cline { 2 - 4 } & set 5 & 296 & 80 bulls and 1276 cows \\
\hline
\end{tabular}

${ }^{*} \mathrm{CH}$ reference datasets; when using joint reference datasets for prediction 4398 Nordic Holstein bulls were included.
Kb (on BTA10). As shown in Figure 1, most distances between adjacent markers were less than $100 \mathrm{~Kb}$. However, 2.55\% adjacent SNP had large distance gaps (larger than $200 \mathrm{~Kb})$. This indicates that SNP used in the current study were not evenly distributed over the bovine chromosomes.

Figure 2 presents the distribution of $r_{L D}^{2}$ of adjacent SNP pairs in the $\mathrm{CH}$ and $\mathrm{NH}$ populations. The LD between adjacent SNP pairs was generally small. The proportion of adjacent SNP pairs which had an $r_{L D}^{2}<0.01$ was $15.2 \%$ for $\mathrm{CH}$ and $17.6 \%$ for $\mathrm{NH}$; the proportion with an $r_{L D}^{2}<0.1$ was $52.81 \%$ for $\mathrm{CH}$ and $51.72 \%$ for $\mathrm{NH}$. These results indicate that the SNP markers did not effectively capture all the QTL affecting a trait, since most adjacent SNP pairs were in weak LD. The mean $r_{L D}^{2}$ of adjacent SNP pairs within a chromosome ranged from 0.16 to 0.24 in $\mathrm{CH}$ and from 0.16 to 0.25 in $\mathrm{NH}$. The mean $r_{L D}^{2}$ across all chromosomes was 0.20 in $\mathrm{CH}$ and 0.21 in $\mathrm{NH}$. The consistency of LD between the $\mathrm{CH}$ and $\mathrm{NH}$ populations was high (Table 2). The correlation of $r_{L D}$ of adjacent SNP ranged from 0.95 to 0.98 across all chromosomes, with a mean of 0.97 at an average marker distance of $60 \mathrm{~Kb}$.

The mean $r_{L D}^{2}$ in the $\mathrm{CH}$ and $\mathrm{NH}$ populations was similar to the degree of LD reported for the Holstein populations in Germany [17], in the Netherlands, Australia and New Zealand [12], and in North America [18]. The consistency of LD between the $\mathrm{CH}$ and $\mathrm{NH}$ populations agreed with the consistency of LD between the Dutch and Australian Holstein bulls reported in [12] and was in line with the development of the $\mathrm{CH}$ population. The first dairy cattle imported in China came from Europe in the 1870's [19] and since then, Holstein cattle have continuously been imported from Europe, Japan and North America. The imported Holstein bulls were crossed with local yellow cattle, and the crossbred cows were continuously back-crossed with the imported Holstein bulls [20]. The resulting crossed black and white dairy cattle were officially named Chinese Holsteins in 1992. Currently, most of the Holstein bulls found in China were imported from worldwide in the form of embryos or live cattle. Besides, the $\mathrm{NH}$ population has also exchanged genetic material with the United States, the Netherlands, Germany and other countries. The genomic relationship matrix showed that some $\mathrm{CH}$ bulls could be full-sibs or half-sibs of the NH bulls. Based on these data, it can be inferred that the Chinese Holstein population is genetically close to European and North American Holstein cattle.

\section{Genomic prediction}

Reliabilities of GEBV for the $48 \mathrm{CH}$ test bulls that had no genotyped daughters and the $1572 \mathrm{CH}$ cows in the test dataset with the five-fold cross-validation are presented in Table 3. When the $\mathrm{CH}$ cattle were used as the reference 
Table 2 Distance and linkage disequilibrium $\left(r_{\mathrm{LD}}^{2}\right)$ of adjacent SNP for each Bos taurus autosome (BTA)

\begin{tabular}{|c|c|c|c|c|c|c|}
\hline \multirow[t]{2}{*}{ BTA } & \multirow{2}{*}{$\begin{array}{l}\text { Length } \\
\text { (Mb) }\end{array}$} & \multirow{2}{*}{$\begin{array}{l}\text { Number } \\
\text { of SNP }\end{array}$} & \multirow{2}{*}{$\begin{array}{c}\text { Mean } \\
\text { distance } \\
\text { (Kb) }\end{array}$} & \multicolumn{2}{|c|}{ Mean $r_{\mathrm{LD}}^{2}$} & \multirow[t]{2}{*}{ Consistency } \\
\hline & & & & Chinese Holstein & Nordic Holstein & \\
\hline 1 & 160.89 & 2729 & 58.98 & 0.22 & 0.23 & 0.97 \\
\hline 2 & 138.92 & 2221 & 62.58 & 0.22 & 0.22 & 0.98 \\
\hline 3 & 125.41 & 2067 & 60.70 & 0.21 & 0.22 & 0.97 \\
\hline 4 & 120.39 & 2031 & 59.30 & 0.20 & 0.21 & 0.97 \\
\hline 5 & 124.59 & 1740 & 71.64 & 0.21 & 0.21 & 0.97 \\
\hline 6 & 119.01 & 2099 & 56.73 & 0.22 & 0.23 & 0.97 \\
\hline 7 & 112.37 & 1809 & 62.15 & 0.22 & 0.24 & 0.98 \\
\hline 8 & 115.59 & 1940 & 59.61 & 0.21 & 0.22 & 0.98 \\
\hline 9 & 104.64 & 1639 & 63.88 & 0.20 & 0.21 & 0.97 \\
\hline 10 & 103.09 & 1752 & 58.87 & 0.22 & 0.22 & 0.97 \\
\hline 11 & 106.97 & 1858 & 57.61 & 0.21 & 0.21 & 0.97 \\
\hline 12 & 89.17 & 1338 & 66.70 & 0.19 & 0.20 & 0.97 \\
\hline 13 & 83.84 & 1434 & 58.50 & 0.22 & 0.22 & 0.97 \\
\hline 14 & 83.15 & 1409 & 59.06 & 0.24 & 0.25 & 0.98 \\
\hline 15 & 83.81 & 1371 & 61.17 & 0.18 & 0.19 & 0.96 \\
\hline 16 & 80.09 & 1283 & 62.47 & 0.22 & 0.23 & 0.98 \\
\hline 17 & 74.89 & 1324 & 56.60 & 0.19 & 0.20 & 0.97 \\
\hline 18 & 65.16 & 1101 & 59.24 & 0.19 & 0.19 & 0.96 \\
\hline 19 & 62.83 & 1131 & 55.60 & 0.18 & 0.20 & 0.97 \\
\hline 20 & 72.00 & 1314 & 54.84 & 0.20 & 0.21 & 0.97 \\
\hline 21 & 68.46 & 1110 & 61.73 & 0.21 & 0.22 & 0.97 \\
\hline 22 & 60.14 & 1047 & 57.50 & 0.19 & 0.19 & 0.97 \\
\hline 23 & 51.73 & 895 & 57.86 & 0.17 & 0.18 & 0.97 \\
\hline 24 & 62.77 & 1053 & 59.67 & 0.21 & 0.21 & 0.98 \\
\hline 25 & 43.23 & 825 & 52.46 & 0.19 & 0.20 & 0.96 \\
\hline 26 & 50.95 & 871 & 58.57 & 0.19 & 0.20 & 0.97 \\
\hline 27 & 45.33 & 801 & 56.67 & 0.16 & 0.16 & 0.96 \\
\hline 28 & 46.06 & 783 & 58.90 & 0.16 & 0.17 & 0.95 \\
\hline 29 & 50.53 & 863 & 58.62 & 0.17 & 0.18 & 0.97 \\
\hline Mean & $2506.00^{2}$ & $41838^{2}$ & 59.59 & 0.20 & 0.21 & 0.97 \\
\hline
\end{tabular}

${ }^{1}$ The correlation of $r_{L D}$ of adjacent SNP pairs between two populations; ${ }^{2}$ Sum over 29 autosomes.

data, the reliabilities of GEBV were 0.16 for $\mathrm{CH}$ test bulls and 0.14 for $\mathrm{CH}$ cows, averaged over the three traits. When using the joint reference dataset, the average reliabilities increased to 0.45 for $\mathrm{CH}$ bulls and to 0.21 for $\mathrm{CH}$ cows. Based on data from the Dairy Association of China, the reliability of a conventional pedigree index (calculated as $0.5 *$ sire $\mathrm{EBV}+0.25 *$ maternal grandsire EBV) in the $\mathrm{CH}$ population was 0.12 for milk production traits. Thus, the reliabilities of genomic predictions based on the $\mathrm{CH}$ reference population were of similar magnitude as the reliabilities of a pedigree index, and the genomic predictions based on the joint reference population gave much higher reliabilities than those based on a pedigree index.

The low reliabilities of genomic predictions using the $\mathrm{CH}$ reference dataset alone suggested that the information in the $\mathrm{CH}$ reference dataset, which mainly contained cows, was insufficient. According to Goddard [2], the reliability of GEBV for different sizes of reference population and different heritabilities of traits can be predicted as:

$$
E\left(r_{G E B V}^{2}\right)=1-\frac{\lambda}{2 N \sqrt{a}} \times \log \frac{1+a+2 \sqrt{a}}{1+a-2 \sqrt{a}}
$$

where $N$ is the number of individuals in the reference population and $a=1+2 \lambda / N$. According to Hayes et al. [21], $\lambda=M_{e} k / h^{2}, M_{e}=2 N_{e} L$, and $k=1 / \log (2 N e)$, where $N_{e}$ is the effective population size and $L$ is the length of the genome in Morgans. Using the above formula, the reliability of GEBV based on the $\mathrm{CH}$ reference dataset was expected to be 0.175 , assuming $L=30, N e=100, N=1500$ 


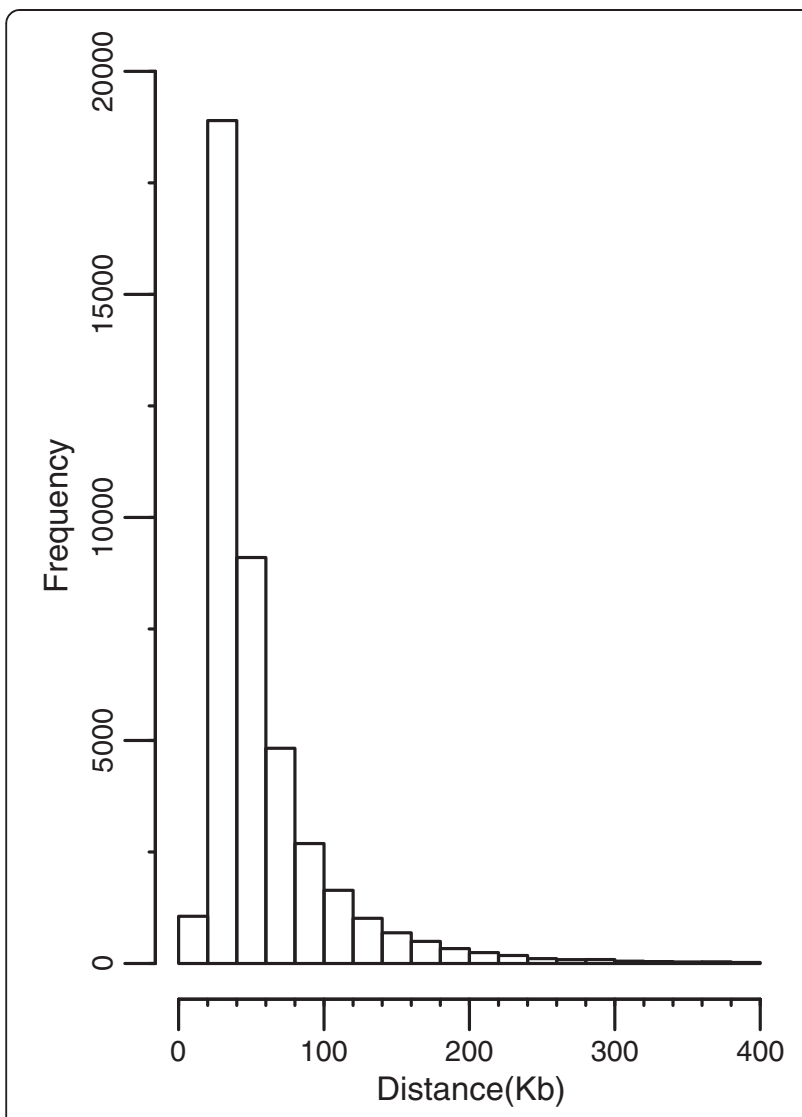

Figure 1 Frequency distribution of distances between adjacent SNP pairs. and $r_{D R P}^{2}=0.50$. The reliabilities obtained from the validation procedures were consistent with these expected reliabilities. The results indicate that the size of the $\mathrm{CH}$ reference population needs to be increased in order to increase the reliability of genomic predictions.

Dairy cattle reference populations usually comprise progeny-tested bulls to maximize the information from each genotyped individual. In some countries or in some cattle populations where the number of progeny-tested bulls is small, one solution is to include cows in the reference population. In order to evaluate the value of adding cows to the reference dataset, an additional analysis was performed using a $\mathrm{CH}$ reference dataset from which $50 \%$ of the cows were deleted. The reliabilities of GEBV for the $\mathrm{CH}$ bulls using the reduced $\mathrm{CH}$ reference dataset decreased to $0.09,0.03$ and 0.05 for milk yield, fat yield and protein yield, respectively. This indirectly demonstrates that it is feasible to use cows as reference animals for genomic prediction, when the number of available progeny-tested bulls is not sufficient. A simulation study by Mc Hugh et al. [22] also suggested that genomic information from cows could greatly increase genetic gain and accuracy of male selection. To increase the size of the cow reference population at low cost, a good alternative would be to genotype cows using a low density chip like the Bovine LD $(7 \mathrm{~K})$ and then impute the genotypes for the $54 \mathrm{~K}$ panel.

The joint reference dataset greatly improved the reliability of genomic predictions for the $\mathrm{CH}$ cattle. The reliabilities of GEBV for $\mathrm{CH}$ bulls based on the joint reference dataset were close to those for $\mathrm{NH}$ bulls based on the Nordic reference data [23]. Several studies have reported that the reliability of genomic prediction can be

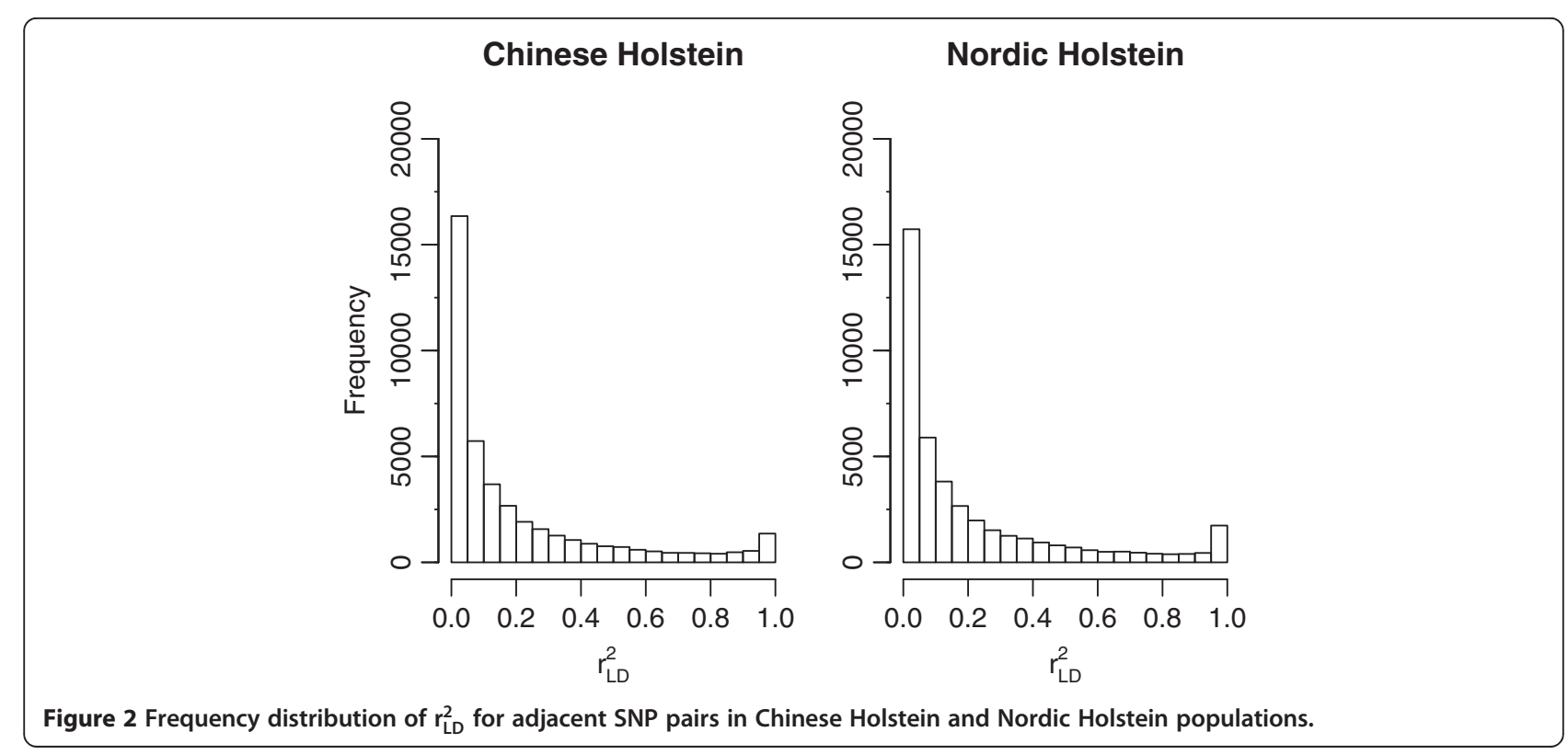


Table 3 Reliabilities of GEBV of Chinese Holstein (CH) bulls and cows in the test populations when using the $\mathrm{CH}$ or the joint reference population

\begin{tabular}{lllll}
\hline Categories & \multicolumn{1}{c}{ Traits } & & Reliabilities of prediction \\
\cline { 2 - 5 } & & Using CH reference & Using joint reference & Increase \\
\hline CH bulls & Milk yield & 0.22 & 0.51 & 0.47 \\
\cline { 2 - 5 } & Fat yield & 0.15 & 0.36 & 0.29 \\
\cline { 2 - 5 } & Protein yield & 0.11 & 0.45 & 0.25 \\
\cline { 2 - 5 } & Average & 0.16 & 0.26 & 0.29 \\
\hline CH cows & Milk yield & 0.15 & 0.17 & 0.05 \\
\cline { 2 - 5 } & Fat yield & 0.12 & 0.20 & 0.05 \\
\cline { 2 - 5 } & Protein yield & 0.15 & 0.21 & 0.07 \\
\cline { 2 - 5 } & Average & 0.14 & & 0.11 \\
\hline
\end{tabular}

increased by using a joint reference dataset that includes reference animals from other populations. The reliabilities of GEBV increased by $10 \%$ on average when four European Holstein populations were combined into a reference dataset, compared to when only one national population was used as the reference population [5]. Reliabilities of genomic prediction for Canadian Holstein bulls increased by $6 \%$ on average when about 3000 foreign bulls were included in the reference dataset [4], and by $7 \%$ when all North American sires were included [24]. Reliabilities were 2.6\% higher for Holstein and 3.2\% higher for Brown Swiss cattle when 3593 foreign Holstein and 732 foreign Brown Swiss animals were included in the reference dataset of the USA domestic prediction [6].

With the joint reference dataset, reliabilities of genomic predictions improved more for $\mathrm{CH}$ bulls than for $\mathrm{CH}$ cows i.e. by 2.3 fold for $\mathrm{CH}$ bulls and only by 1.7 fold for $\mathrm{CH}$ cows. This is due to the fact that $\mathrm{CH}$ bulls have a closer relationship with $\mathrm{NH}$ bulls than the $\mathrm{CH}$ cows do. Among the $48 \mathrm{CH}$ test bulls, 14 bulls had a genomic relationship with one or more $\mathrm{NH}$ bull in the range from 0.45 to 0.56 . However, no $\mathrm{CH}$ cow had this level of relationship with any $\mathrm{NH}$ bull. Moreover, among the $48 \mathrm{CH}$ test bulls, 33 $(68.75 \%)$ had a genomic relationship greater than 0.2 with at least one $\mathrm{NH}$ bull (with 15.5 bulls on average). Among the $1572 \mathrm{CH}$ cows, only 459 (29.2\%) had a genomic relationship greater than 0.2 with an $\mathrm{NH}$ bull (with 1.3 bulls on average). Many previous studies reported that the existence of a close relationship between test animals and reference animals increased the reliability of genomic predictions for the test animals [25-27].

To avoid overestimation of the reliability of GEBV, 19 $\mathrm{CH}$ bulls were excluded from the test dataset because they were highly related to 13 bulls in the reference population. In the five-fold cross-validation for the $\mathrm{CH}$ cows, two or three half-sib families were randomly assigned to a singletest dataset, instead of randomly choosing individuals. This was done to avoid overestimation of the reliability of GEBV when animals in the test dataset have a large group of half-sibs in the reference dataset. Moreover, genetic trend can increase the correlation between GEBV and DRP if the birth years of the animals in the test dataset cover a wide range. Therefore, in the validation for the $\mathrm{CH}$ bulls, the correlation between GEBV and DRP was calculated after correcting for genetic trend. When ignoring this correction, the validation reliabilities of genomic predictions for $\mathrm{CH}$ bulls using the joint reference dataset were unrealistically high at $0.69,0.54$ and 0.60 for milk yield, fat yield and protein yield, respectively.

In the current study, when using the joint reference dataset, genomic predictions were estimated using a twotrait model, in which the same biological trait was considered to be a different trait in the $\mathrm{CH}$ and $\mathrm{NH}$ populations. The reason for using a two-trait model, instead of a singletrait model, was that the DRP had different scales in the two populations due to the use of a standardization procedure in the $\mathrm{NH}$ population. The two-trait model also accounts for the presence of any genotype by environment interactions. When genotypes of bulls from three foreign countries were included in the USA domestic predictions, multi-trait methods were not more accurate than the single-trait model for Holstein cattle, but gave higher reliabilities (1.4\% higher on average) for Brown Swiss cattle [6]. The authors suggested that this could be due to lower genetic correlations of traits between Brown Swiss populations. Using the two-trait GBLUP model, the estimated genetic correlations between the $\mathrm{CH}$ and $\mathrm{NH}$ populations were $0.85,0.70$ and 0.75 for milk yield, fat yield and protein yield respectively, which were much lower than the value of the consistency of LD of neighboring markers, which was 0.97 between the two populations. Assuming that the consistency of LD was appropriate to represent the genetic associations between different populations, its clear difference with the estimated genetic correlations suggests the existence of a large genotype by environment interaction between China and Nordic countries.

\section{Conclusions}

The consistency of LD is very high between the $\mathrm{CH}$ and $\mathrm{NH}$ populations, indicating a high level of genetic 
similarity between the two populations. Genomic prediction for $\mathrm{CH}$ cattle can be greatly improved using a joint reference dataset that includes $\mathrm{CH}$ and $\mathrm{NH}$ cattle. In order to obtain satisfactory reliabilities of genomic predictions for $\mathrm{CH}$ cattle, it is necessary to increase the size of the $\mathrm{CH}$ reference population or to include foreign Holstein cattle in the reference population.

\section{Competing interests}

The authors declare that they have no competing interests.

\section{Authors' contributions}

LZ performed the analysis and drafted the manuscript with help from GS. XD prepared Chinese Holstein data and helped in analyzing the data. GS, MSL, QZ and YW conceived and designed the study. MSL and GS helped in interpreting results. All the authors read, revised and approved the final manuscript.

\section{Acknowledgments}

This work was performed in the projects "Genomic Selection-From function to efficient utilization in cattle breeding (grant no. 3405-10-0137)", funded under Green Development and Demonstration Programme (Denmark); "Combining Nordic and Chinese reference populations for genomic selection", funded by VikingGenetics (Denmark); the Chinese National '948' Project (2011-G2A); The National Natural Science Foundation of China (31272418); Ph.D. Programs Foundation of Ministry of Education of China (20110008110001); and China earmarks fund for CARS-37.

\section{Author details}

Received: 16 October 2012 Accepted: 6 March 2013

Published: 21 March 2013

\section{References}

1. Meuwissen THE, Hayes BJ, Goddard ME: Prediction of total genetic value using genome-wide dense marker maps. Genetics 2001, 157:1819-1829.

2. Goddard M: Genomic selection: prediction of accuracy and maximisation of long term response. Genetica 2009, 136:245-257.

3. Daetwyler HD, Villanueva B, Woolliams JA: Accuracy of predicting the genetic risk of disease using a genome-wide approach. PLoS One 2008, 3:e3395.

4. Schenkel FS, Sargolzaei M, Kistemaker G, Jansen GB, Sullivan P, Van Doormaal BJ, VanRaden PM, Wiggans GR: Reliability of genomic evaluation of Holstein cattle in Canada. Interbull Bull 2009, 39:51-57.

5. Lund MS, de Roos APW, de Vries AG, Druet T, Ducrocq V, Fritz S, Guillaume F, Guldbrandtsen B, Liu ZT, Reents R, Schrooten C, Seefried F, Su GS: A common reference population from four European Holstein populations increases reliability of genomic predictions. Genet Sel Evol 2011, 43:43.

6. VanRaden PM, Olson KM, Null DJ, Sargolzaei MMW, van Kaam JBCHM: Reliability increases from combining 50,000- and 777,000-marker genotypes from four countries. Interbull Bull 2012, 46:75-79.

7. Jorjani H, Jakobsen J, Nilforooshan MA, Hjerpe E, Zumbach B, Palucci V, Dürr $\mathrm{J}$ : Genomic evaluation of BSW populations interGenomics: results and deliverables. Interbull Bull 2011, 43:5-8.

8. Brondum RF, Rius-Vilarrasa E, Stranden I, Su G, Guldbrandtsen B, Fikse WF, Lund MS: Reliabilities of genomic prediction using combined reference data of the Nordic Red dairy cattle populations. J Dairy Sci 2011, 94:4700-4707.

9. Guo G, Zhou L, Liu L, Li D, Zhang SL, Liu JF, Ding XD, Zhang Y, Wang YC, Zhang Q, Zhang Y: Parentage inference with single nucleotide polymorphism markers in the Chinese Holstein in Beijing. Xu Mu Shou Yi Xue Bao 2011, 43:44-49.

10. Browning SR, Browning BL: Rapid and accurate haplotype phasing and missing-data inference for whole-genome association studies by use of localized haplotype clustering. Am J Hum Genet 2007, 81:1084-1097.

11. Hill WG, Robertson A: Linkage disequilibrium in finite populations. Theor Appl Genet 1968, 38:226-231.

12. de Roos APW, Hayes BJ, Spelman RJ, Goddard ME: Linkage disequilibrium and persistence of phase in Holstein-Friesian, Jersey and Angus cattle. Genetics 2008, 179:1503-1512.
13. Hayes BJ, Goddard ME: Technical note: Prediction of breeding values using marker-derived relationship matrices. J Anim Sci 2008, 86:2089-2092.

14. VanRaden PM: Efficient methods to compute genomic predictions. J Dairy Sci 2008, 91:4414-4423.

15. Su G, Madsen P, Nielsen US, Mantysaari EA, Aamand GP, Christensen OF, Lund MS: Genomic prediction for Nordic Red Cattle using one-step and selection index blending. J Dairy Sci 2012, 95:909-917.

16. Madsen $P$, Jensen J: A users guide to DMU, version 6, release 5.0. Tjele: University of Aarhus, Faculty of Agricultural Science; 2010.

17. Qanbari S, Pimentel ECG, Tetens J, Thaller G, Lichtner P, Sharifi AR, Simianer $\mathrm{H}$ : The pattern of linkage disequilibrium in German Holstein cattle. Anim Genet 2010, 41:346-356.

18. Sargolzaei M, Schenkel FS, Jansen GB, Schaeffer LR: Extent of linkage disequilibrium in Holstein cattle in North America. J Dairy Sci 2008, 91:2106-2117.

19. Qiu H, Qin Z, Chen Y, Wang A: Bovine breeds in China. Shanghai: Shanghai Scientific \& Technical Publishers: 1988

20. Qin ZY: The breeding of Chinese Holstein. China Dairy 2001, 10:26-27.

21. Hayes BJ, Visscher PM, Goddard ME: Increased accuracy of artificial selection by using the realized relationship matrix. Genet Res 2009, 91:47-60.

22. Mc Hugh N, Meuwissen THE, Cromie AR, Sonesson AK: Use of female information in dairy cattle genomic breeding programs. J Dairy Sci 2011, 94:4109-4118

23. Gao HD, Christensen OF, Madsen P, Nielsen US, Zhang Y, Lund MS, Su GS: Comparison on genomic predictions using three GBLUP methods and two single-step blending methods in the Nordic Holstein population. Genet Sel Evol 2012, 44:8.

24. Muir B, Van Doormaal B, Kistemaker G: International genomic cooperation North American perspective. Interbull Bull 2010, 41:71-76.

25. Clark SA, Hickey JM, Daetwyler HD, van der Werf JHJ: The importance of information on relatives for the prediction of genomic breeding values and the implications for the makeup of reference data sets in livestock breeding schemes. Genet Sel Evol 2012, 44:4.

26. Habier D, Fernando RL, Dekkers JCM: The impact of genetic relationship information on genome-assisted breeding values. Genetics 2007, 177:2389-2397.

27. Pszczola M, Strabel T, Mulder HA, Calus MPL: Reliability of direct genomic values for animals with different relationships within and to the reference population. J Dairy Sci 2012, 95:389-400.

doi:10.1186/1297-9686-45-7

Cite this article as: Zhou et al: Consistency of linkage disequilibrium between Chinese and Nordic Holsteins and genomic prediction for Chinese Holsteins using a joint reference population. Genetics Selection Evolution 2013 45:7.

\section{Submit your next manuscript to BioMed Central and take full advantage of:}

- Convenient online submission

- Thorough peer review

- No space constraints or color figure charges

- Immediate publication on acceptance

- Inclusion in PubMed, CAS, Scopus and Google Scholar

- Research which is freely available for redistribution 